\title{
Spectroscopy of Be Acceptor Ground State in GaAs/AlGaAs Heterostructure
}

\author{
J. ŁusAkowski ${ }^{a, *}$, R. BuczKo ${ }^{b}$, M. SAKowicz ${ }^{a}$, \\ K.J. Friedland ${ }^{c}, \mathrm{R} \cdot \mathrm{HeY}^{c}$ and K. $\mathrm{PlOOG}^{c}$ \\ ${ }^{a}$ Institute of Experimental Physics, Warsaw University \\ Hoża 69, 00-681 Warsaw, Poland \\ ${ }^{b}$ Institute of Physics, Polish Academy of Sciences \\ al. Lotników 32/46, 02-668 Warsaw, Poland \\ ${ }^{c}$ Paul-Drude-Institut für Festkörperelektronik \\ Hausvogteiplatz 5-7, 10117 Berlin, Germany
}

Photoluminescence measurements were carried out on Be $\delta$-doped $\mathrm{GaAs} / \mathrm{Al}_{0.33} \mathrm{Ga}_{0.67} \mathrm{As}$ heterostructure at $1.6 \mathrm{~K}$ in magnetic fields $(B)$ up to $4 \mathrm{~T}$. Luminescence originating from recombination of a two-dimensional electron gas and photoexcited holes localized on Be acceptors was analyzed. The degree of circular polarization $\left(\gamma_{\mathrm{C}}\right)$ of the luminescence from fully occupied Landau levels was determined as a function of $B$ and the two-dimensional electron gas concentration, $n_{s}$. At $B$ constant, $\gamma_{\mathrm{C}}$ decreased with the increase in $n_{s}$. The intensity of the optical transition considered was calculated with taking into account $s$ - and $d$-like parts of the acceptor envelope function. It is shown that the presence of the $d$-like part explains the observed $\gamma_{\mathrm{C}}\left(n_{s}\right)$ dependence quantitatively. This shows that polarization spectroscopy on acceptor $\delta$-doped heterostructures enables one to test experimentally the contribution of the $L>0$ component of the envelope in a shallow acceptor description.

PACS numbers: 71.70.Ej, 78.67.De

\section{Introduction}

The paper describes experiments carried out on acceptor $\delta$-doped GaAs/AlGaAs heterostructures and a theoretical analysis of experimental results. The process investigated is a radiative recombination of a two-dimensional electron gas (2DEG) with photoexcited holes localized on acceptors in the $\delta$-layer. An analysis of the degree of circular polarization $\left(\gamma_{\mathrm{C}}\right)$ of this transition as a function

*corresponding author; e-mail: jerzy.lusakowski@fuw.edu.pl 
of the magnetic field $(B)$ allowed us to investigate spin polarization of a 2DEG in the regime of the integer and fractional quantum Hall effects [1]. An interpretation of experiments presented in [1] was based on a model in which the ground state of a shallow acceptor in GaAs was described by an $s$-like envelope function (the " $L=0$ model"). According to this model, $\gamma_{\mathrm{C}}$ depends only on the magnetic field and the temperature $(T)$ : the magnetic field splits electron and hole levels which are populated according to a thermal distribution at given $T$. Within this approach, properties of a 2DEG could be analyzed [1] because the properties of holes localized on acceptors were assumed to be known.

According to our experimental studies, $\gamma_{\mathrm{C}}$ depends also on the concentration of the 2DEG $\left(n_{s}\right)$ at $B$ and $T$ constant. To explain this effect, we analyze the influence of a $d$-like part of the acceptor envelope on $\gamma_{\mathrm{C}}$ (the " $L=0,2$ " model) and we show that the consideration of it describes the observed $\gamma_{\mathrm{C}}\left(n_{s}\right)$ dependence.

\section{Experiment and results}

The sample under investigation was a high quality $\mathrm{GaAs} / \mathrm{Al}_{0.33} \mathrm{Ga}_{0.67} \mathrm{As}$ heterostructure grown on semi-insulating GaAs substrate. The GaAs layer of about $1 \mu \mathrm{m}$ above 50 periods of $5 \mathrm{~nm} / 5 \mathrm{~nm}$ GaAs/AlAs superlattice contains unintentional acceptors at a concentration smaller than $10^{14} \mathrm{~cm}^{-3}$. The AlGaAs barrier comprises an undoped $45 \mathrm{~nm}$ thick AlGaAs spacer and a uniformly Si-doped $35 \mathrm{~nm}$ thick AlGaAs layer; the doping level amounts to $10^{18} \mathrm{~cm}^{-3}$. The $\delta$-layer of Be atoms with the concentration of $10^{9} \mathrm{~cm}^{-2}$ was introduced into the GaAs channel at the distance $z_{0}=30 \mathrm{~nm}$ away from the GaAs/AlGaAs interface. The barrier was covered with a $15 \mathrm{~nm}$ thick GaAs cap layer.

The measurements were carried out at $1.6 \mathrm{~K}$ in magnetic fields up to $4 \mathrm{~T}$. The luminescence passed through a $\lambda / 4$ plate followed by a quartz linear polarizer. The circular polarizations were separated by turning the $\lambda / 4$ plate. The luminescence was analyzed by a spectrometer supplied with a CCD camera. A semi-transparent $\mathrm{Au}$ gate electrode and an ohmic contact were fabricated on the sample surface and the concentration of the 2DEG was tuned by polarizing the gate in the backward direction. $n_{s}$ was estimated by determination of the magnetic field, $B_{\nu=k}$, where $k=1$ or 2 and $\nu$ is the filling factor, at which the luminescence from the $N=k$ Landau levels (LLs) disappears. Then $n_{s}=k B_{\nu=k} /(h / e)$, where $e$ is the electron charge and $h$ is the Planck constant. Other experimental details can be found in $[2]$.

An example of the magnetic field evolution of the luminescence spectrum is shown in Fig. 1. With an increase in $B$, the number of populated LLs of the first electrical subband decreases as their degeneracy grows. The analysis of the polarization of the luminescence starts with a deconvolution of each spectrum into separate Lorentzian peaks corresponding to pairs of LLs. We subtract from the total spectrum Lorentzians corresponding to the second electrical subband and the highest in energy pair of LLs of the first electrical subband. In such a way 


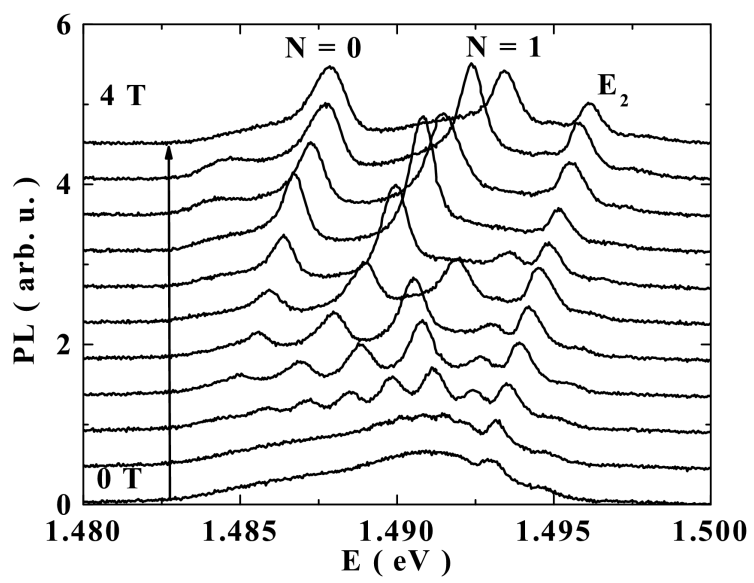

Fig. 1. An example of the evolution of the luminescence spectrum with the magnetic field $B$ from 0 to 4 T, every $0.4 \mathrm{~T}$ (bottom to top). LLs of the ground electrical subband and the peak corresponding to the first excited $\left(E_{2}\right)$ subband are visible. Two lowest LLs are labeled with their number, $N$. In this case, the $N=2$ peak disappears at $B_{\nu=4} \approx 2.6 \mathrm{~T}$ which corresponds to $n_{s} \approx 2.4 \times 10^{11} \mathrm{~cm}^{-2}$. The spectra are vertically shifted for better presentation.

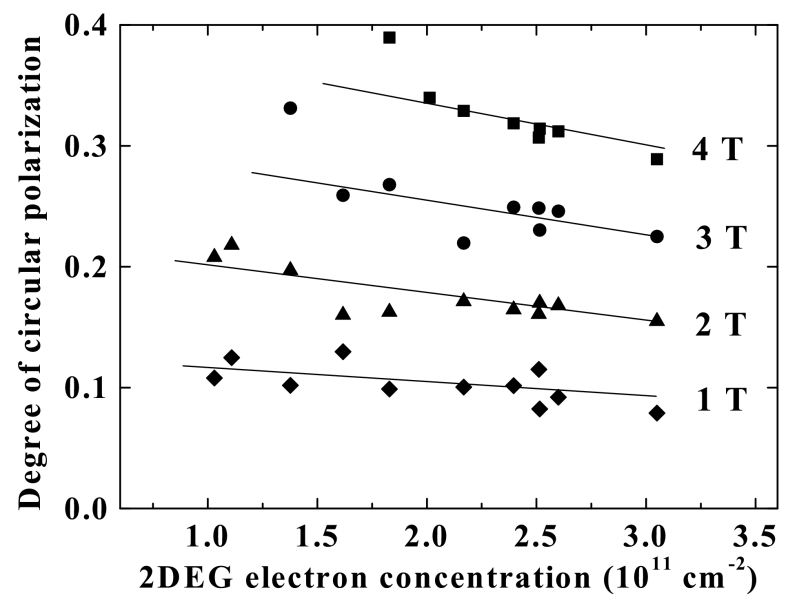

Fig. 2. Measured $\gamma_{\mathrm{C}}\left(n_{s}\right)$ dependence for $1 \mathrm{~T}$ (diamonds), $2 \mathrm{~T}$ (triangles), $3 \mathrm{~T}$ (circles), and $4 \mathrm{~T}$ (squares). Solid lines are the results of model calculations (shifted down to take into account depolarization by the experimental system).

we obtain a part of the spectrum which corresponds to an equal number of LLs occupied with spin-up and spin-down electrons, i.e., to totally unpolarized electron gas. In this way, we exclude from considerations possible spin effects. The area of that part of the spectrum $\left(I_{\sigma^{+}}\right.$or $I_{\sigma^{-}}$for $\sigma^{+}$and $\sigma^{-}$polarization, respectively $)$ 
is used to calculate $\gamma_{\mathrm{C}}=\left(I_{\sigma^{-}}-I_{\sigma^{+}}\right) /\left(I_{\sigma^{-}}+I_{\sigma^{+}}\right)$. This procedure allows us to determine $\gamma_{\mathrm{C}}$ at given $B$ for different $n_{s}$. The results are presented in Fig. 2 and show that $\gamma_{\mathrm{C}}$ depends on $n_{s}$ at $B$ constant.

\section{Theoretical analysis and conclusions}

The interpretation of the $\gamma_{\mathrm{C}}\left(n_{s}\right)$ dependence observed is based on calculations of matrix elements of the optical transition considered within the following model. The ground state of the system is represented by an ionized shallow acceptor located at $z_{0}=30 \mathrm{~nm}$ from the heterostructure interface. The excited state is given by an electron which is created in one of the Landau levels and a hole in the shallow acceptor ground state. We use an approximate description of the excited state neglecting the Coulomb interaction between the electron and the neutral acceptor. We apply the dipole approximation for optical transitions and the one-electron description of electronic bands. Then, a matrix element of the transition is proportional to $\left\langle\psi_{i}\left|\hat{p}_{1, \pm 1}\right| \chi_{j}\right\rangle$, where $\left|\psi_{i}\right\rangle$ and $\left|\chi_{j}\right\rangle$ are the one-electron Landau and acceptor states, respectively, and subscripts $i$ and $j$ represent appropriate sets of quantum numbers. The electronic $\chi_{j}(\boldsymbol{r})$ wave function is obtained by the time reversal of the bound hole wave function. The component $+1(-1)$ of the momentum operator $\left(\hat{p}_{1, \pm 1}=\mp\left(\hat{p}_{x} \pm \mathrm{i} \hat{p}_{y}\right)\right)$ is used according to the circular polarization $\sigma^{+}\left(\sigma^{-}\right)$of the emitted photon. The effective mass approximation has been used for the description of electronic wave functions. We also assume that the electron effective mass in the 2D conduction subband is isotropic and we use the spherical approximation of the shallow acceptor in the bulk GaAs, as described in the paper of Baldereschi and Lipari [3].

The calculations are described in detail in [2]. The results of the calculations are shown in Fig. 2 with solid lines. One can clearly see that the proposed model describes quantitatively a $\gamma_{\mathrm{C}}\left(n_{s}\right)$ dependence. The disregard of the $L=2$ part of the acceptor envelope leads to $\gamma_{\mathrm{C}}$ that does not depend on $n_{s}$, contrary to experimental data.

The spherical approximation used in the present paper is necessary and sufficient to describe experimental data. A more detailed description of the acceptor is given by the cubic approximation [4] which takes into account an infinite series of spherical harmonics with even $L$. Then, transitions between any pair of electron and hole levels would be allowed in both polarizations. We expect, however, that the intensity of transitions, additional to those considered in the present paper, is negligible since it results from higher order perturbations to the $L=0,2$ model.

In conclusion, polarized magnetoluminescence experiments on a GaAs/ $\mathrm{AlGaAs}$ heterostructure doped with Be acceptor $\delta$-layer in the GaAs channel were carried out as a function of $n_{s}$. The degree of circular polarization of the luminescence, originating from transitions between the 2DEG and photoexcited holes bound to Be acceptors, was measured and found to decrease with the increase in $n_{s}$. We showed that the observed $\gamma_{\mathrm{C}}\left(n_{s}\right)$ dependence results from the presence 
of the $L=2$ part in the acceptor envelope wave function. We thus show that the interband polarization spectroscopy in acceptor $\delta$-doped heterostructures allows one to investigate the ground state of a shallow acceptors in bulk GaAs.

\section{References}

[1] I.V. Kukushkin, V.B. Timofeev Adv. Phys. 45, 147 (1996).

[2] J. Łusakowski, R. Buczko, M. Sakowicz, K.J. Friedland, R. Hey, K. Ploog, J. Phys., Condens. Matter 19, 236205 (2007).

[3] A. Baldereschi, N.O. Lipari, Phys. Rev. B 8, 2697 (1973).

[4] A. Baldereschi, N.O. Lipari, Phys. Rev. B 9, 1525 (1974). 